STEM CELL BIOLOGY

\title{
Transbronchial biopsies provide longitudinal evidence for epithelial chimerism in children following sex mismatched lung transplantation
}

\author{
H Spencer, D Rampling, P Aurora, D Bonnet, S L Hart, A Jaffé
}

Thorax 2005;60:60-62. doi: 10.1136/thx.2004.029678

See end of article for authors' affiliations

......................

Correspondence to: Dr A Jaffé, Consultant and Honorary Senior Lecturer in Respiratory Research, Portex Anaesthesia, Intensive Therapy and Respiratory Medicine Unit, Institute of Child Health and Great Ormond Street Hospital for Children NHS Trust, Great Ormond Street, London WCIN 3JH, UK; A.Jaffe@ich.ucl. ac.uk

Received 7 June 2004 Accepted 30 September 2004
Background: Recent reports have shown evidence of host derived parenchymal engraftment in several human allografts including the lung, leading to speculation that stem cell therapy may be useful for lung repair in diseases such as cystic fibrosis (CF). To date, previous studies have looked at single surgical or autopsy specimens and no longitudinal studies have been reported. The aim of this study was to assess whether transbronchial biopsies could be used to study the time course of chimerism following lung transplantation.

Methods: Specimens of archived transbronchial lung biopsies from five time points taken for clinical purposes from two boys who had received a sex mismatched heart-lung transplant for end stage CF were examined. Sections were dual stained for cytokeratin (epithelium) and a mixture of leucocyte common antigen and CD68 for inflammatory cells. Co-localisation of cells containing a $Y$ chromosome was confirmed by fluorescent in situ hybridisation.

Results: Evidence of chimerism was found in up to $6.6 \%$ of epithelial cells in bronchial (median $1.4 \%$ (range 0-6.6)) and alveolar (median 3.6\% (range 2.3-5.5) tissue without apparent evidence of fusion. This engraftment was seen as early as 3 weeks and remained relatively constant up to 37 months.

Conclusions: This study has demonstrated proof of principle for long term chimerism in lung epithelium. Transbronchial biopsies may provide a new method for studying the kinetics of stem cell engraftment in the lung.
G ene therapy by topical delivery to the airway of patients with cystic fibrosis (CF) has been disappointing to date. Recent reports have led to speculation that stem cells might provide a more effective vehicle for delivery of gene therapy. ${ }^{1}$ Experimental studies in murine models have provided evidence that bone marrow stem cells can differentiate into types I and II pneumocytes and bronchial epithelial cells. ${ }^{23}$ Human studies following haemopoietic stem cell transplantation (HSCT) have shown evidence of donor derived cells (chimerism) in the lung $(n=4)$ by examining necroscopic and surgical lung biopsy tissue. ${ }^{45} \mathrm{~A}$ recent report has demonstrated chimerism as early as 4 days and up to 7 years in seven archived human lung allografts explanted because of organ failure. ${ }^{6}$ Long term engraftment in a single subject has never been shown. The aim of this study was to investigate the utility of transbronchial biopsies to assess long term engraftment in the lung following sex mismatched lung transplantation.

\section{METHODS \\ Subjects}

Of the 80 lung transplants performed at Great Ormond Street Hospital for Children over the past 15 years, 12 boys have received a female heart-lung or lung transplant. Only two patients had multiple biopsies available for more than 1 year following transplantation and these were selected for examination. Transbronchial biopsies were performed for clinical purposes using a $1.9 \mathrm{~mm}$ diameter biopsy forceps (EndoJaw, Olympus Corporation, Tokyo, Japan) passed down the suction channel of a flexible bronchoscope under fluoroscopic guidance. Transbronchial biopsy tissues from a female and a male patient following a sex matched lung transplant were used as controls. Ethical approval for the study was obtained from the Great Ormond Street Hospital for Children and Institute of Child Health ethics committee.

\section{Immunohistochemistry and fluorescent in situ hybridisation (FISH)}

Sections of $4 \mu \mathrm{m}$ thickness were cut from formalin fixed, paraffin embedded transbronchial biopsy tissue. To discriminate host derived lung cells from inflammatory cells, the section underwent dual immunostaining for cytokeratin (MNF116) at a dilution of 1:500 to demonstrate epithelial cells (DAKO UK Ltd) visualised with diaminobenzidine, followed by staining with a mixture of leucocyte common antigen (LCA) at a dilution of 1:300 (CD45) and CD68 (a macrophage marker) at a dilution of 1:100 (both DAKO UK Ltd) visualised with fuschin (fig IC and G). ${ }^{2}$ Fluorescent in situ hybridisation (FISH) for the $\mathrm{X}$ and Y chromosome (Vysis, CEP X SpectrumOrange/CEP YspectrumGreen, Abbott Laboratories Ltd, UK) was performed on the same section (fig 1D and H). ${ }^{4}$ Nuclei were counterstained with $4^{\prime} 6$ diamidine 2-phenylindole dihydrochloride (DAPI, Vector Laboratories Ltd, UK).

\section{Imaging and analysis}

A Leica TCS SP confocal microscope with UV laser was used to take images of five random areas of epithelium and five random areas of alveoli at $\times 63$ oil magnification from each time point. Areas in which lung epithelium were obscured by clumps of white cells were avoided. To create each three dimensional image a stack of 17 sections was made. To demonstrate immunohistochemical staining the same areas were examined with a Zeiss Axioskop 2 light microscope at $\times 63$ oil magnification and images were taken with a digital camera. At least 1-5 (median 2) confocal images were matched to the immunostained images and a 

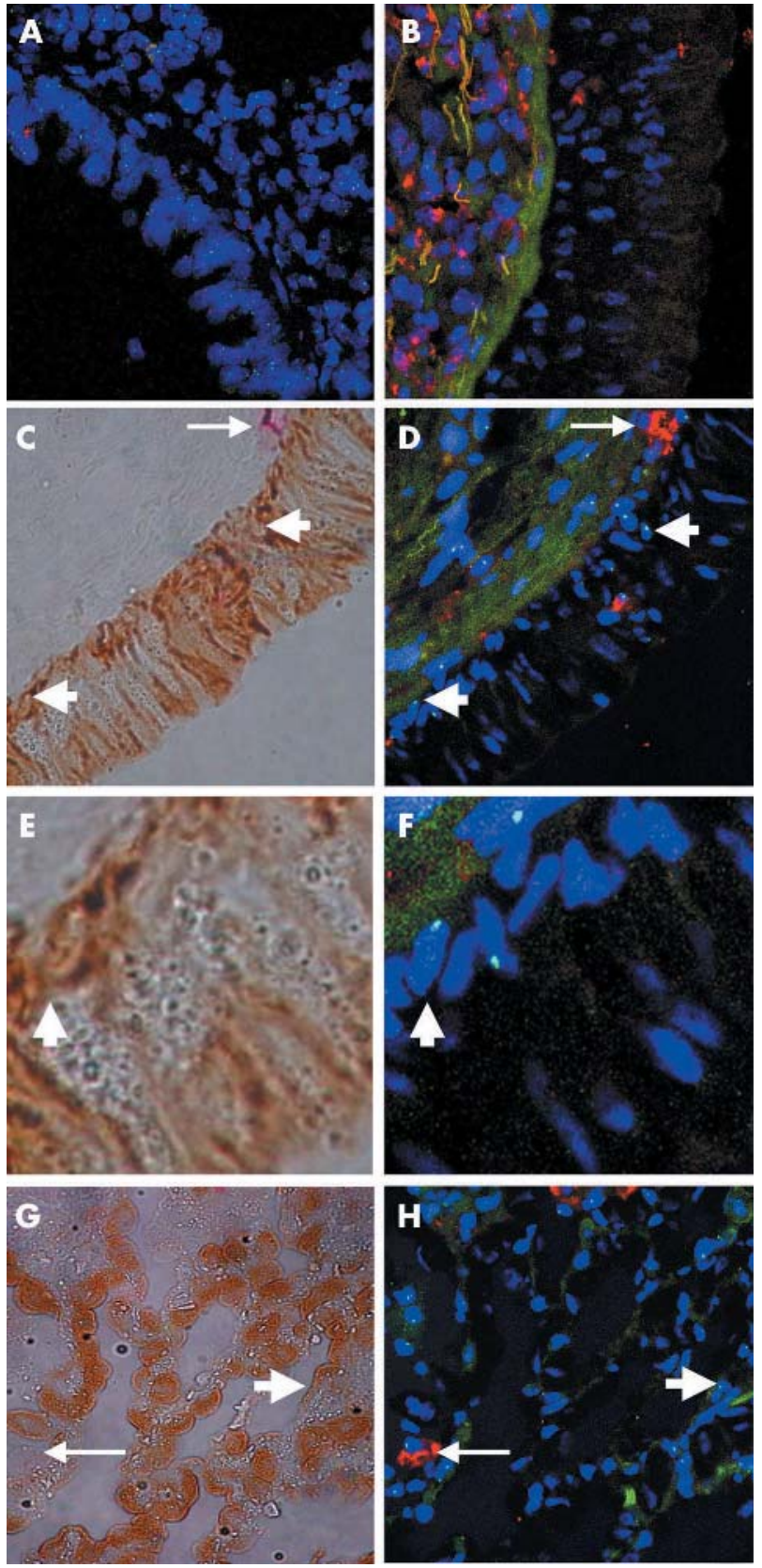

Figure 1 Detection of host derived epithelial cells in transbronchial biopsy specimens. Fluorescent in situ hybridisation (FISH) staining of (A) male and (B) female controls showing $Y$ chromosomes (green dots) and $X$ chromosomes (smaller red dots). Light microscope images of (C) bronchial tissue (patient 1 at 14 weeks) and (G) alveolar tissue (patient 2 at 4 weeks) from male patients following sex mismatched heart-lung transplantation showing cytokeratin staining of bronchial epithelium (brown DAB stain). Epithelial cells which co-stain for $Y$ chromosome are present (thick arrows). Inflammatory cells positive for a mixture of CD45 and CD68 (red Fuschin stain) are shown (thin arrows) (magnification $\times 63$ ). Confocal images of same sections ( $D$ and $H$ ) examined by FISH for $\mathrm{X}$ chromosome and $\mathrm{Y}$ chromosome and nuclear DAPI stain (blue) (magnification $\times 63$ ). CD45 and CD68 positive cells also show as red stain under fluorescence (thin arrows). Host derived Y FISH positive cells which also co-stain for cytokeratin (thick arrows) are present in the epithelial layer. (E) and (F) An epithelial cytokeratin positive cell costained with a $Y$ chromosome at higher magnification $(\times 200)$.

picture overlay was performed using Adobe Photoshop to show co-localisation of cytokeratin and Y chromosome. The total number of nuclei, $\mathrm{Y}$ positive nuclei, $\mathrm{Y}$ positive white cells (CD45 or CD68 positive), and Y positive epithelial cells (cytokeratin positive) were counted manually and Y positive cells expressed as a percentage of the total number of nuclei examined. ${ }^{1}$

\section{RESULTS}

Alveolar and bronchial areas of transbronchial biopsy specimens were examined at five time points (fig 2) from two boys with $\mathrm{CF}$ aged 11 and 13 years. Both patients had routine immunosuppression with azathioprine, cyclosporin $\mathrm{A}$, and prednisolone. Patient 1 (fig 2A and B) had no episodes of rejection. Patient 2 (fig $2 \mathrm{C}$ and $\mathrm{D}$ ) had multiple episodes of rejection from day 8 onwards which was treated with methylprednisolone.

The median (range) percentage of all lung cell nuclei containing a Y chromosome was 8.2\% (1.9-17.3\%) (absolute number 39 (7-94), total nuclei examined 470 (120-635)). Of those positive for a Y chromosome, $1.4 \%(0-8.3 \%)$ were inflammatory cells (absolute number $4(0-12)$, total nuclei examined $470(120-635))$. In the alveolar region 3.6\% (2.3$5.5 \%$ ) of epithelial cells were Y positive (absolute number 16 (2-22), total nuclei examined 352.5 (52-562), fig 2A and C) while, in the bronchial region, $1.4 \%(0-6.6 \%)$ of epithelial cells were Y positive (absolute number $3.5(0-8)$, total nuclei examined 254 (57-445), fig $2 \mathrm{~B}$ and $\mathrm{D})$. A number of $\mathrm{Y}$ positive cells (absolute number 39 (7-94), total nuclei examined 470 (120-635)) did not co-stain for either cytokeratin or CD45 or CD68.

To investigate the possibility of cell fusion accounting for $\mathrm{Y}$ positive cells, confocal microscopy was used to create a three dimensional picture to determine the number of chromosomes within a single nucleus. We found all nuclei were diploid. The female control biopsy specimen was entirely negative for the $\mathrm{Y}$ chromosome. In the male control biopsy only $67 \%$ of Y chromosomes could be visualised due to partial sampling of nuclei. This finding suggests that we may have underestimated the amount of engraftment in lung tissue by approximately $30 \%$.

\section{DISCUSSION}

Host derived epithelial cells were found in the alveolar and bronchial epithelium as early as 3 weeks and remained relatively stable up to 37 months following lung transplantation, suggesting that there was no clonal expansion. This lack of increase in engraftment over time may limit treatment where repopulation of the lung with healthy tissue is required. However, we speculate that stem cell therapy may potentially be of therapeutic benefit in CF where replacement of as little as $6 \%$ of healthy cells is enough to correct the chloride channel defect. ${ }^{7}$

Interestingly, we saw similar engraftment rates in both patients and did not see any significant increase in engraftment in the patient who had multiple episodes of rejection and presumed ongoing injury. This is contrary to recent studies showing that induction of lung injury increases localisation of bone marrow cells in the lung. ${ }^{236}$ It is possible that the immunosuppressive treatment which patient 2 received for rejection prevented this from occurring. In addition, the immunosuppression given before biopsy may also explain why we did not see an increase in white cells in the biopsy specimens taken at times of rejection (4 weeks: mild to moderate rejection; 16 weeks: moderate rejection), although a more likely explanation is that we focused on areas of lung tissue in which inflammatory cells did not obscure the vision of epithelial cells in order to increase the chances of detecting epithelial chimerism. We presume that host stem cells or progenitor cells replace lung cells in the transplanted lung, which are lost as a consequence of the various injury processes associated with lung transplantation. We speculate that the small fluctuation in the numbers of $Y$ 

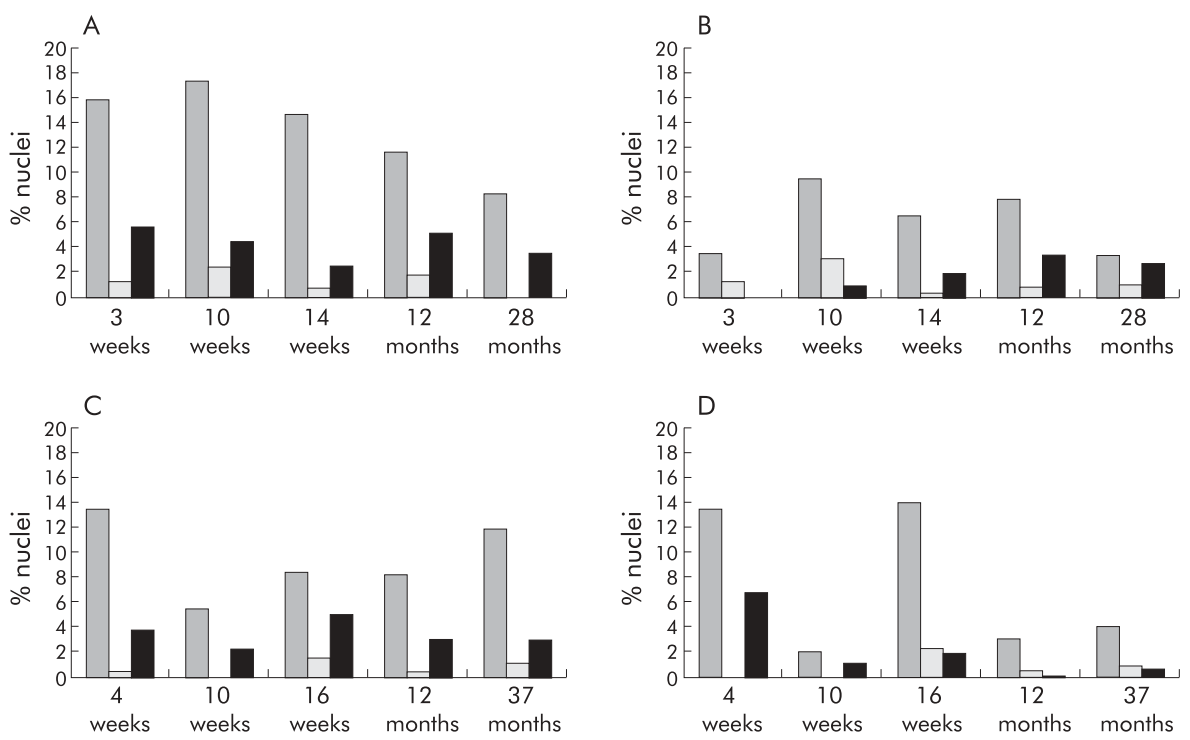

Figure 2 Long term epithelial engraftment in two boys after sex mismatched heart-lung transplantation assessed at five time points. (A) Alveolar region and (B) bronchial region of patient 1. (C) Alveolar region and (D) bronchial region of patient 2. Darker shaded columns show the percentage of total $Y$ positive cells, lighter shaded columns show the percentage of $Y$ positive and CD45 or CD68 positive cells, and solid black columns show the percentage of $Y$ positive and cytokeratin positive cells.

positive cells over time (fig 2) is likely to be the result of limitations of the technique rather than a change in repeated engraftment with cell turnover.

One limitation of our study is that we have not shown evidence of epithelial cell function. In addition, there were a large number of Y positive cells which did not stain as white cells or epithelial cells and may represent endothelial, fibroblast, or unlabelled inflammatory cells. The amount of available lung tissue obtained from transbronchial biopsies limited the number of staining procedures carried out. We were also limited by the number of suitable patients available, as in previous studies. ${ }^{4-6}$ We suggest that future studies should include taking multiple transbronchial biopsy samples for research purposes to address the questions that we have been unable to answer using archived tissue.

In summary, we have shown, for the first time, longitudinal evidence of host derived epithelial cells in transplanted lungs without apparent evidence of cell fusion. Transbronchial biopsy specimens are potentially a viable tissue resource for investigating the kinetics of stem cell engraftment in the lung and may potentially be useful in future stem cell therapeutic trials. These findings lead to speculation about the use of stem cells in the repair and regeneration of damaged lung tissue in diseases such as CF.

\section{ACKNOWLEDGEMENTS}

The authors are grateful to Steve Bottoms and Robin McNulty for guidance with immunohistochemistry and to Pauline Whitmore for providing patient details.

\section{Authors' affiliations}

H Spencer, A Jaffé, P Aurora, Portex Anaesthesia, Intensive Therapy and Respiratory Medicine Unit, Institute of Child Health and Great Ormond Street Hospital for Children NHS Trust, London, UK
D Rampling, Histopathology Department, Camelia Botnar Laboratories, Great Ormond Street Hospital for Children NHS Trust, London, UK

P Aurora, Department of Lung Transplantation, Great Ormond Street Hospital for Children NHS Trust, London, UK

D Bonnet, Cancer Research UK, London Research Institute, London, UK S L Hart, Molecular Immunology Unit, Institute of Child Health, University College London, London, UK

This study was made possible by a grant from the Cystic Fibrosis Trust. Conflict of interest: none declared.

H Spencer, A Jaffe, S Hart and D Bonnet designed the study. H Spencer and D Rampling did the experimental work. P Aurora carried out the transbronchial biopsies. All researchers contributed to the preparation of and approved the report.

This work was presented at the 27th European Cystic Fibrosis Conference 2004.

\section{REFERENCES}

1 Grove JE, Lutzko C, Priller J, et al. Marrow-derived cells as vehicles for delivery of gene therapy to pulmonary epithelium. Am J Respir Cell Mol Biol 2002; 27:645-51.

2 Krause DS, Theise ND, Collector MI, et al. Multi-organ, multi-lineage engraftment by a single bone marrow-derived stem cell. Cell 2001;105:369-77.

3 Kotton DN, Ma BY, Cardoso WV, et al. Bone marrow-derived cells as progenitors of lung alveolar epithelium. Development 2001;128:5181-8.

4 Suratt BT, Cool CD, Seris AE, et al. Human pulmonary chimerism after haemopoietic stem cell transplantation. Am J Respir Crit Care Med 2003; 168:318-22.

5 Mattsson J, Jansson M, Wernerson A, et al. Lung epithelial cells and type II pneumocytes of donor origin after allogeneic hematopoietic stem cell transplantation. Transplantation 2004;78:154-7.

6 Kleeberger W, Versmold A, Rothamel T, et al. Increased chimerism of bronchial and alveolar epithelium in human lung allografts undergoing chronic injury. Am J Pathol 2003; 162:1487-94.

7 Johnson LG, Olsen JC, Sarkadi B, et al. Efficiency of gene transfer for restoration of normal airway epithelial function in cystic fibrosis. Nat Genet 1992; $2: 21-5$ 